\title{
- Avaliação da importância do sistema porta-renal em papagaios (Amazona aestiva)
}

- Evaluation of the importance of the porta-renal system in parrots (Amazona aestiva)

*Faculdade de Medicina Veterinária e Zootecnia

Departamento de Cirurgia e

Anestesiologia Veterinária - UNESP

Distrito Rubiāo Júnior

CEP 18.618-000 - Botucatu - SP

e-mail: neca.cruz@mailcity.com

* MariângelaLozano Cruz ${ }^{1}$-CRMV-SP- n $^{0} 8211$

Stelio PaccaLoureiro Luna ${ }^{1}$-CRMV-SP- ${ }^{0}{ }^{\circ} 4420$

Gladys Bastos Castro ${ }^{1}$ - CRMV-SP- $\mathrm{n}^{0} 1103$

Flávio Massone ${ }^{1}$ - CRMV-SP- n $^{0} 263$

FranciscoJosé TeixeiraNeto ${ }^{1}$-CRMV-SP- n $^{0} 7305$

1 - Docentes da Disciplina de Anestesiologia, FMVZ - UNESP/Botucatu.

\section{RESUMO}

Quando um medicamento é administrado na musculatura da coxa de uma ave, é absorvido por capilares da região, o fluxo sangüíneo atinge a veia ilíaca externa, que possui uma válvula porta renal na altura do rim. Esta válvula é controlada pelo sistema nervoso autônomo, podendo estar aberta ou fechada, dependendo de estímulo simpático ou parassimpático, respectivamente. Quando aberta, o fluxo sangüíneo vai para a veia cava através da veia renal e atinge a circulação sistêmica. Porém, quando a válvula está fechada, o fluxo sangüíneo vai para o parênquima renal adicionando-se ao sangue arterial e, conseqüientemente, o medicamento administrado pode ser eliminado pelo rim, antes de atingir a circulação sistêmica. $\mathrm{O}$ objetivo desse experimento foi avaliar a eficácia da quetamina administrada na musculatura da coxa ou do peito em papagaios. Foram utilizados 6 papagaios (Amazona aestiva), os quais foram anestesiados com $30 \mathrm{mg} / \mathrm{kg}$ de quetamina pela via intramuscular no peito ( $\mathrm{G} 1, \mathrm{n}=6)$ ou na coxa ( $\mathrm{G} 2 \mathrm{n}=6)$, utilizando-se os mesmos animais nos dois grupos. Foram avaliados os seguintes parâmetros: tempo da aplicação do fármaco ao decúbito, período de latência, período de duração anestésica e período de recuperação. Não foi constatada nenhuma diferença estatística significativa entre os grupos, porém, em G2, dois animais foram descartados da análise estatística por não manifestarem sinais clínicos de anestesia, provavelmente por uma subdose relativa, isto é, parte da quetamina pode ter sido eliminada com a participação do sistema porta renal, antes de atingir a circulação sistêmica, o que sugere que a musculatura da coxa deve ser evitada quando se utilizam fármacos que são excretados "in-natura" pelos rins.

Palavras-chave: psitacídeo, papagaio, sistema porta renal, quetamina. 


\section{Introdução}

$\underline{0}$ papagaio-verdadeiro (Amazona aestiva) é um psitacídeo, comumente encontrado no Brasil (SOUZA, 1987). É uma espécie freqüente em parques zoológicos, criadouros e também é utilizada como animal de estimação. Ocasionalmente, os animais precisam ser anestesiados para exames clínicos ou intervenções cirúrgicas.

Como nos rins dos mamíferos, os néfrons das aves possuem a capacidade de filtração, excreção (secreção) e reabsorção de substâncias. O túbulo cortical dos néfrons em aves tem a vantagem do suplemento sangüíneo pelo sistema porta-renal (MORILD et al., 1985) (Figura 1). A secreção e reabsorção tubular são alteradas por esse suplemento sangüíneo, o qual é regulado pela abertura ou pelo fechamento da válvula porta renal, localizada bilateralmente na junção da veia ilíaca externa e veia renal (Figura 2).

De acordo com JOHNSON (1979) e WIDEMAN e LAVERTY (1986), o sistema porta renal conduz sangue venoso para o parênquima renal e está relacionado com a excreção de ácido úrico.

Quando um medicamento é administrado na musculatura da coxa de uma ave, é absorvido por capila- res da região, atingindo a veia ilíaca externa, passando pela válvula porta renal na altura do rim (MIRABELLA et al., 1996). Esta válvula é controlada pelo sistema nervoso autônomo, podendo estar aberta ou fechada dependendo de estímulos simpático ou parassimpático, respectivamente. Quando aberta, o fluxo sangüíneo dirige-se para a veia cava através da veia renal e atinge a circulação sistêmica. Porém, quando a válvula está fechada, o fluxo sangüíneo é direcionado para o parênquima renal, adicionando-se ao sangue arterial e, conseqüentemente, o medicamento administrado pode ser eliminado pelo rim antes de atingir a circulação sistêmica (BENNETT e MALMFORMS, 1975).

Durante a contenção física que antecede a anestesia, os animais normalmente estão sob estímulo adrenérgico (simpático) e a válvula porta renal encontra-se aberta (BENNETT e MALMFORMS, 1975); conseqüentemente, o fluxo sangüíneo direciona-se diretamente para a circulação sistêmica através da veia cava.

O objetivo desse experimento foi o de avaliar a eficácia da quetamina administrada na musculatura da coxa ou do peito em papagaios (Amazona aestiva), investigando-se a importância do sistema porta-renal no efeito da droga.

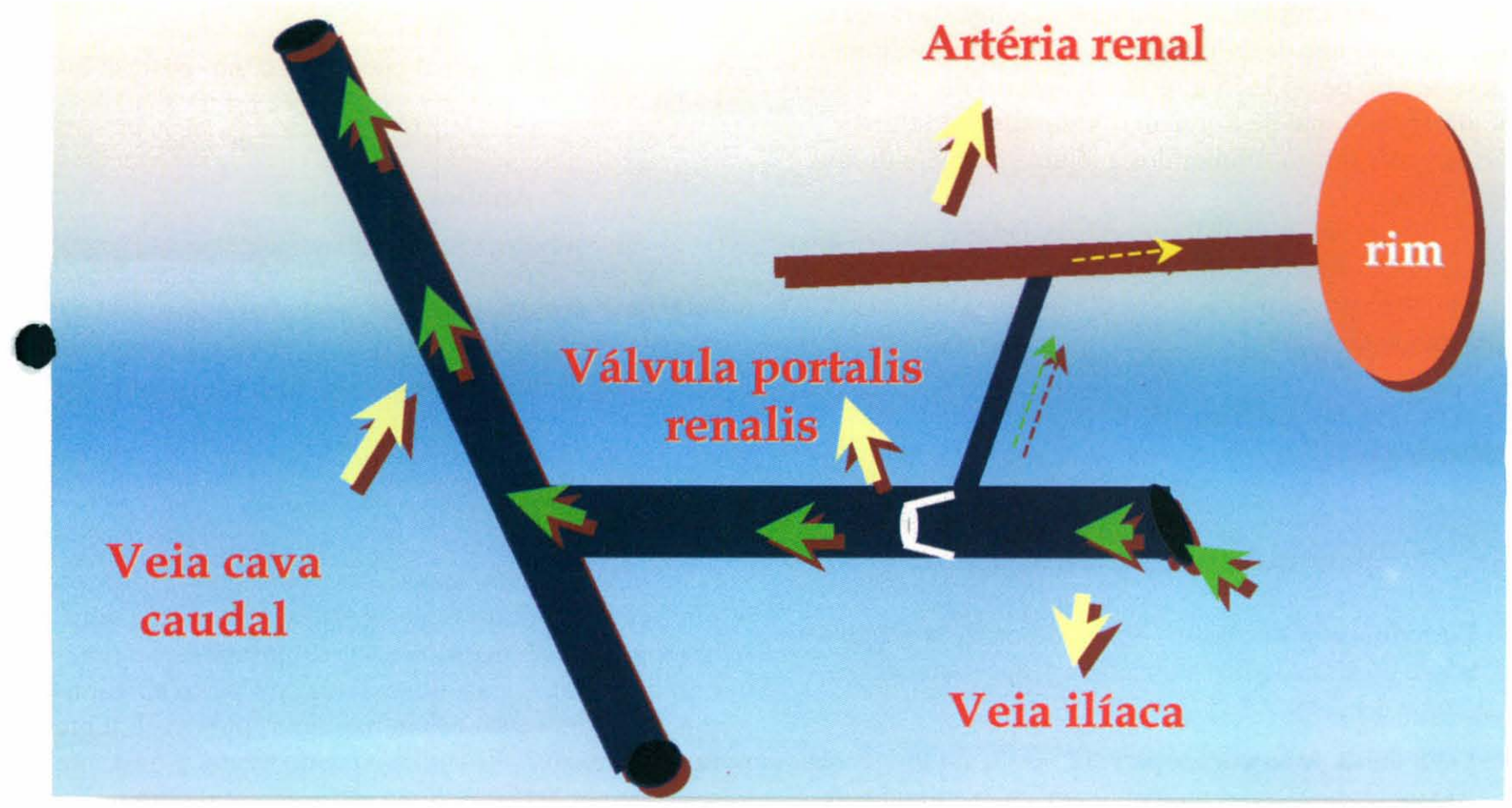

Figura 1. Esquema do sistema porta renal sob ação adrenérgica: válvula aberta. 


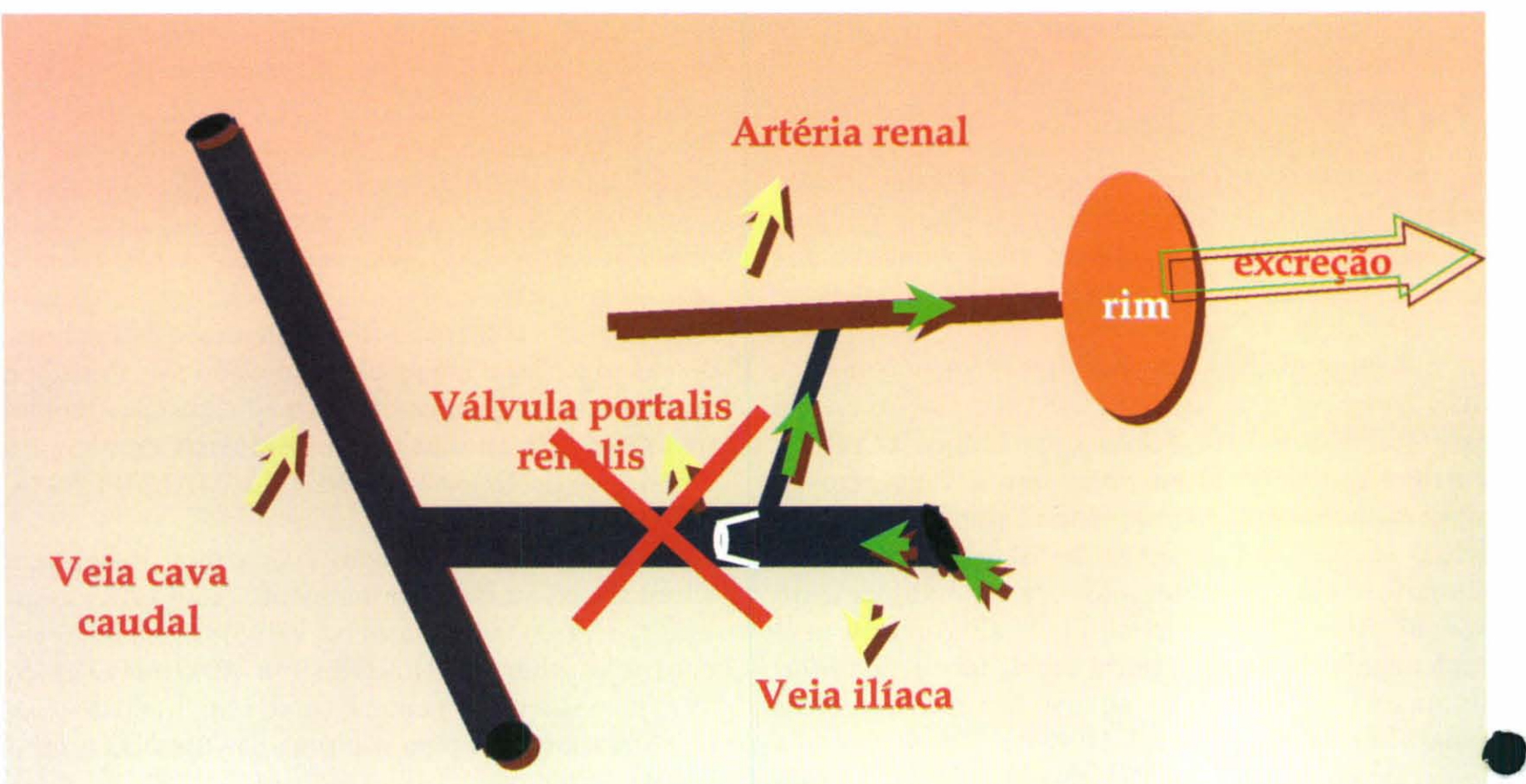

Figura 2. Esquema do sistema porta renal sob ação colinérgica: válvula fechada.

\section{Material e Método}

Foram utilizados 6 papagaios (Amazona aesti$v a$ ), de sexo não definido por sexagem, adultos, com o peso médio de $353 \pm 15 \mathrm{~g}$, procedentes do Parque Zoológico Municipal de Sorocaba "Quinzinho de Barros". Os animais foram submetidos a jejum completo durante 3 horas.

Todos os animais foram anestesiados com $30 \mathrm{mg} /$ $\mathrm{kg}$ de quetamina pela via intramuscular. Foram realizados dois grupos experimentais: G1 $(n=6)$ - a administração da quetamina foi realizada na musculatura peitoral profunda, G2 (n=6) - a administração da quetamina foi realizada na musculatura da coxa. Foram utilizados os mesmos animais nos dois grupos, respeitando-se um intervalo de 10 dias entre cada anestesia.

Foram avaliados os seguintes parâmetros:

- Período até o decúbito - período desde a administração do fármaco até o momento em que o animal apresentou decúbito.

- Período de latência - período desde a administração do fármaco até o momento em que o animal apresentou-se imóvel.
- Período hábil anestésico - período em que o animal apresentou-se imóvel.

- Período de recuperação — desde a administração do fármaco até o animal encontrar-se em posição bipedal.

\section{Análise estatística}

O teste " $t$ " de Student para amostras dependentes foi utilizado para comparar os tempos nos dois grupos. Diferenças foram consideradas significativas quando $\mathrm{p}<0,05$. A Tabela foi expressa em média \pm erro padrão da média.

\section{Resultado e Discussão}

Apesar de não haver diferença estatística no tempo até o decúbito e no período de latência entre os dois grupos (Figuras 3 e 4), os animais que receberam a quetamina na musculatura do peito apresentaram um menor período de latência, provavelmente porque a musculatura profunda do peito é bastante vascularizada, havendo uma absorção mais rápida do fármaco. 


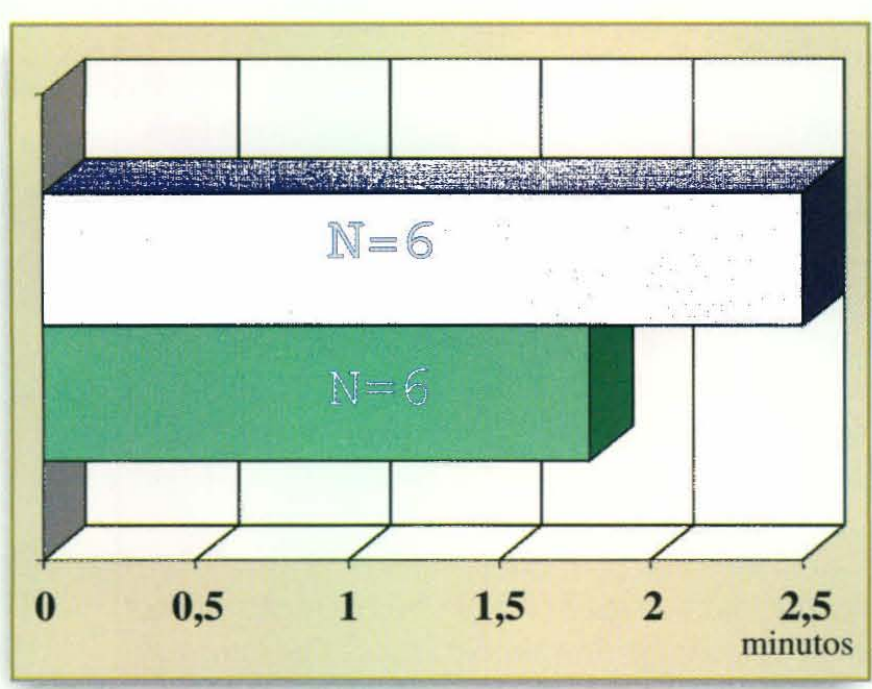

Figura 3. Tempo desde a administração da quetamina até o decúbito em papagaios anestesiados na musculatura da coxa ou do peito.

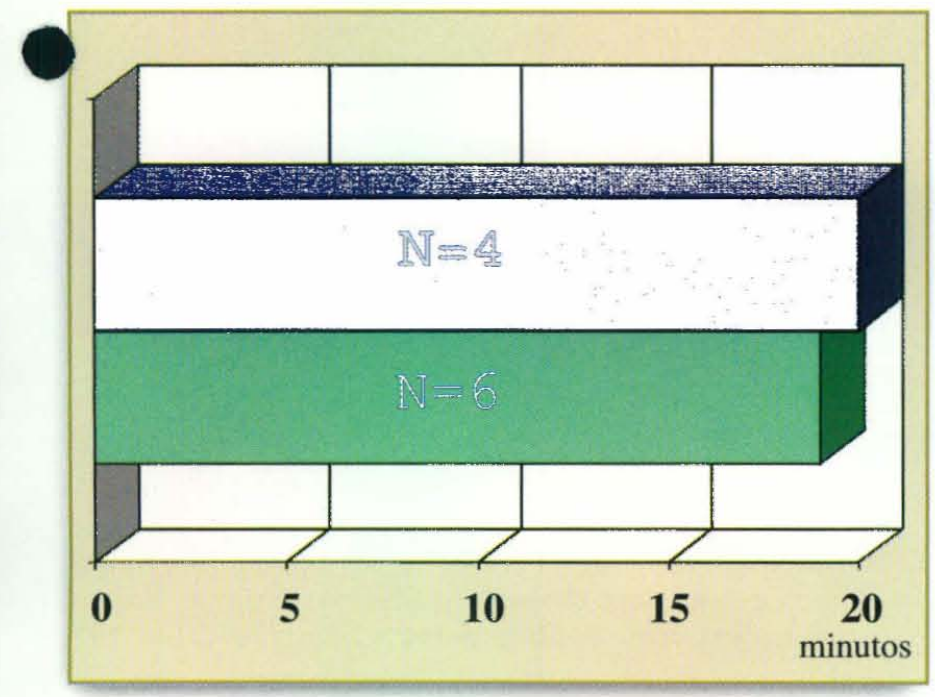

Figura 5. Período hábil anestésico de papagaios anestesiados com quetamina na musculatura da coxa ou do peito.

Conforme exposto na Figuras 5 e 6, não houve diferença estatística na duração da anestesia e no período de recuperação, porém, em G2, dois animais não manifestaram sinais clínicos de anestesia, provavelmente por uma subdose relativa, isto é, parte da quetamina pode ter sido eliminada, com a participação do sistema porta renal, antes de atingir a circulação sistêmica. Isso ocorre quando o animal está sob efeito colinérgico (BENNETT e MALMFORMS, 1975), situação em que a válvula se encontra fechada, e o sangue venoso é direcionado diretamente para os tubulos renais em adição ao sangue arterial. Normalmente, du-

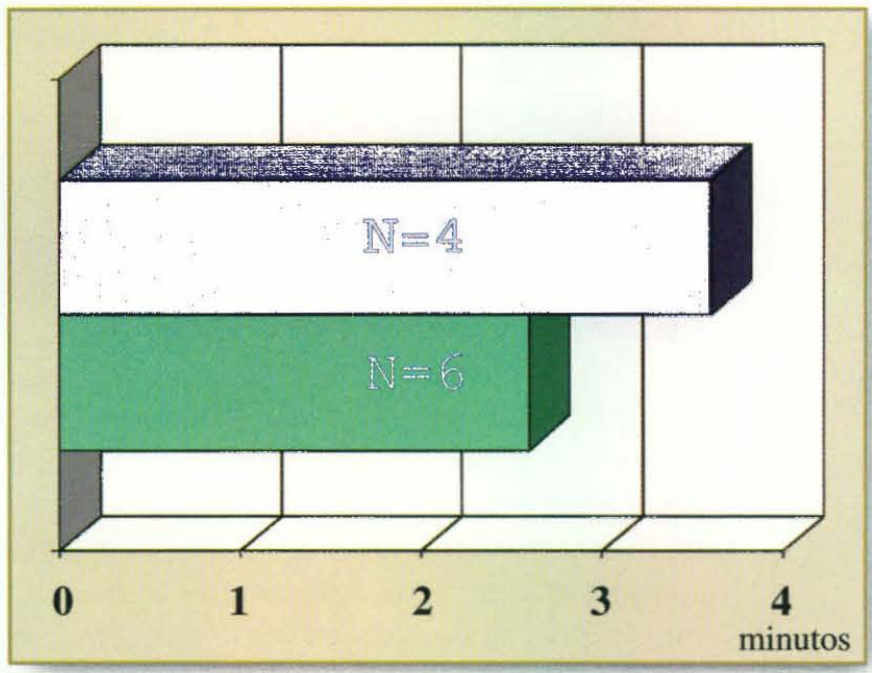

Figura 4. Período de latência, desde a administração da quetamina até total imobilização em papagaios anestesiados com quetamina na musculatura da coxa ou do peito.

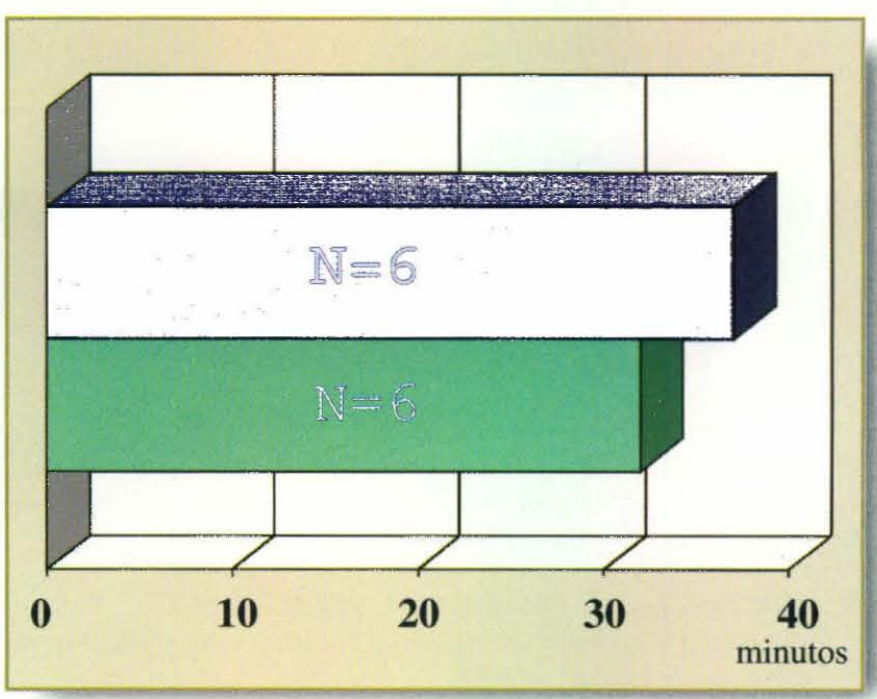

Figura 6. Período de recuperação em papagaios anestesiados com quetamina na musculatura da coxa ou do peito.

rante a contenção física que antecede a anestesia, os animais estão sob efeito adrenérgico, e a válvula porta renal encontra-se aberta (BENNETT e MALMFORS, 1975); conseqüentemente, o fluxo sangüíneo deve se dirigir diretamente para a circulação sistêmica através da veia cava.

Com este experimento, pode-se concluir que se deve evitar a administração de quetamina ou de fármacos que são eliminados "in natura" pelos rins na musculatura da coxa em papagaios, para se evitar o risco de promover uma subdose relativa e efeito inadequado do fármaco. 


\section{SUMMARY}

When injected in the thigh muscles of a bird, the local capillaries absorb the drug and the blood flow goes to the external iliac vein that has a porta-renal valve close to the kidney. Controlled by the autonomous nervous system, this valve can be opened or closed by the sympathetic or parasympathetic stimuli respectively. When opened, the blood flow goes to the renal vein then to the vena cava and reaches the systemic circulation. However, when the valve is closed, the blood flow goes to the renal parenchyma where it is mixed with the arterial blood. Thus, the kidney can excrete the drug that was given before it reaches the systemic circulation. The objective of this study was to evaluate the efficacy of ketamine when injected into the thigh or breast muscle of parrots (Amazona aestiva). Six birds were anesthetized with $30 \mathrm{mg} / \mathrm{kg}$ ketamine by intramuscular injection in the breast $(\mathrm{G} 1, \mathrm{n}=6)$ or thigh $(\mathrm{G} 2, \mathrm{n}=6)$, using the same parrots in both groups. The following parameters were evaluated: time from administration until recumbency, latency period, duration of anesthesia and recovery period. No significant statistical difference was found between the two groups, but two birds of the G2 group were excluded from statistical analysis because they did not show clinical signs of anesthesia. It was probably caused by a relative underdose; that is, part of the ketamine could have been eliminated by the porta-renal system before reaching the systemic circulation. It is suggested that the intramuscular administration of drugs in the thigh should be avoided when the drugs are excreted "in natura" by the kidneys.

Key words: Psittacidae, parrot, porta-renal system, ketamine.

\section{REFERÊNCIAS}

1. BENNETT, T.; MALMFORS, T. Autonomic of renal portal blood flow in the domestic fowl. General pharmacology. v. 6, p. 49. 1975.

2. JOHNSON, O. W.; Urinary organs; In: KING A. S.; MALELLAND, J. (ed): Form and function in birds. New York: Academic Press, 1979. v. 1, P. 183-235.

3. MIRABELLA, N.; ESPOSITO, V.; PELAGALLI, G. V. The valva portalis renalis in the duck (Anas platyrhynchos) Acta Anatomica, v. 157, p. 151-8, 1996.
4. MORILD, I.; BOHLE, A.; CHRISTENSEN, J. A. Structure of the avian kidney. The Anatomical Record, v. 212, p. 33-40, 1985.

5. SOUZA, D. Aves do Brasil. Belo Horizonte: Editora Itatiaia.1987. v. 6 .

6. WIDEMAN Jr., R. F. ; LAVERTY, G. Kidney function in domestic fowl with choronic occlusion of the ureter and caudal renal vein. Poultry Science, v. 65, p. 2148-155, 1986.

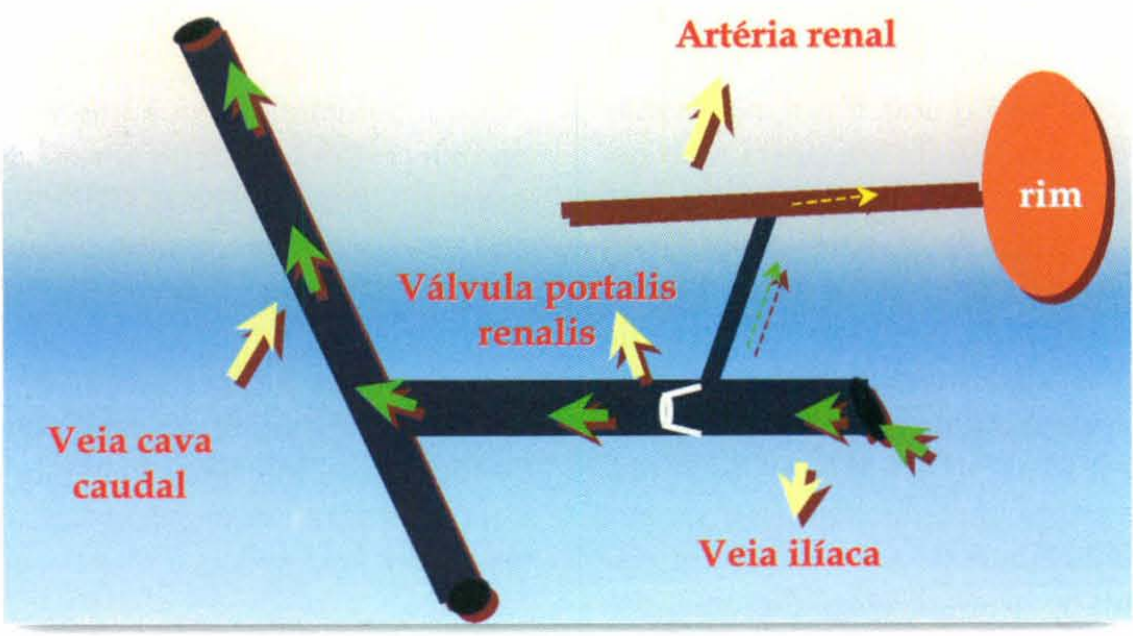

